RUNNING HEAD: Stimulus devaluation by backward inhibition

\author{
Stimulus devaluation by backward inhibition exceeds any \\ emotional impact of cognitive conflict: Evidence from task switching \\ Elizabeth M. Clancy, Greer Gillies, \& Mark J. Fenske \\ Department of Psychology, University of Guelph
}

Words in abstract: 212

Words: 4605 total; 2971 in introduction, results, and discussion

Number of references: 55

\title{
Correspondence:
}

Mark J. Fenske, PhD

Department of Psychology

University of Guelph

Guelph, Ontario N1G 2W1

Email: mfenske@uoguelph.ca

Tel: 519-824-4120 x56411

Acknowledgement: This work was supported by the Natural Science and Engineering Research Council of Canada (Discovery Grant \#401526). 


\begin{abstract}
Ignoring or withholding a response from a stimulus causes it to become affectively devalued. Leading accounts posit that this is due to negative affect elicited by neurocognitive inhibition when it is applied to resolve conflict from distracting or otherwise inappropriate stimulus/response representations. Other research, however, suggests that stimulus/response conflict may itself elicit negative affect and devalue stimuli, raising questions about whether effects previously attributed to inhibition may instead reflect the emotional impact of conflict, per se. To address this, we measured affective ratings of art-like patterns that previously appeared on critical trials of a task-switching paradigm (ABA vs. CBA task sequences) known for its capacity to distinguish behavioural effects of inhibition and conflict. Stimuli from the ABA-sequence experimental condition showing behavioural evidence of backward inhibition (n2 repetition costs) received more negative ratings than those from the CBA-sequence control condition. This stimulus-devaluation effect was not impacted by the level of conflict associated with high uncertainty or low uncertainty about upcoming task order. Moreover, the responsetime index of inhibition was larger on ABA trials in which the associated stimuli later received negative ratings than on trials preceding relatively positive ratings. Inhibition therefore appears to have negative affective consequences that exceed any emotional impact of conflict, with fluctuations in inhibition linked to subsequent stimulus evaluations.
\end{abstract}

Keywords: Task switching; Inhibition; Conflict; Emotion 


\section{Stimulus devaluation by backward inhibition exceeds any emotional impact of cognitive conflict: Evidence from task switching}

Neurocognitive inhibition is critically involved in the control of behavior, attention, memory, and thought (for reviews see Anderson, Bunce \& Barbas, 2016; Bari \& Robbins, 2013; Gaspelin \& Luck, 2018; Munakata, Herd, Chatham, Depue, Banich, \& O’Reilly, 2011). Although predominantly studied for its role in these cognitive-behavioural domains, there is growing evidence that inhibition also has a critical impact on the assessment and representation of emotional and motivational value. For example, several studies have shown that ignoring or withholding a response from a stimulus causes it to become affectively devalued, leading to more negative ratings of such items than of novel items or the targets of attention/response (e.g., Kiss, Raymond, Westoby, Nobre, \& Eimer, 2008; Raymond, Fenske \& Tavassoli, 2003; Veling, Holland \& van Knippenberg, 2008; for reviews see Fenske \& Raymond, 2006; Gollwitzer, Martiny-Huenger, \& Oettingen, 2014; Raymond, 2009). There is a wide range of cognitive domains in which inhibition-based processes are thought to be critical for successful performance, and evidence of a link between inhibition and negative stimulus-linked affect has been found in several of them. This includes devaluation of stimuli associated with behavioural inhibition in Go/No-go tasks (Fenske et al., 2005; Kiss et al., 2008; Veling, Holland, \& Van Knipperberg, 2008) and Stop-signal tasks (Wessel \& O’Doherty, 2014; Wessel, Tonnesen, \& Aron, 2015), with attentional inhibition in visual search (Fenske et al., 2004; Raymond et al., 2005), flanker (Martiny-Huenger, Gollwitzer, \& Oettingen, 2014), attentional blink (Kihara, Yagi, Takeda, \& Kawahara, 2011) and distractor-avoidance tasks (De Vito, Al-Aidroos, \& Fenske, 2017), with long-term memory suppression in Think/No-think (De Vito and Fenske, 2017) and Directed-forgetting tasks (Vivas, Marful, Panagiotidou, \& Bajo, 2016; cf. Janczyk \& Wühr, 2012) and with the inhibition of working memory representations in retro-cue memory- 
search tasks (De Vito \& Fenske, 2018; De Vito, Ferrey, Fenske, \& Al-Aidroos, 2018).

Prominent accounts of such stimulus devaluation findings hypothesize that the effect is due to inhibition immediately eliciting negative affect or otherwise altering the coding of stimulus-linked value when applied to prevent interference from an inappropriate item or its associated response (Clancy, Fiacconi, \& Fenske, 2019; Fragopanagos et al., 2009; Frischen et al., 2012; Raymond, 2009; Veling, Holland, \& van Knippenberg, 2008). However, recent experiments based on Botvinick's (2007) update to conflict-monitoring theory have provided evidence that the conflict between competing stimulus/response representations may itself elicit negative affect (for reviews see Dignath et al., 2020; Dreisbach \& Fischer, 2015) and thereby lead to more negative evaluations of associated stimuli (Damen et al., 2018; Fritz \& Dreisbach, 2013; 2015; Goller et al., 2019). This raises the possibility that the stimulus-devaluation effects previously attributed to inhibition may instead be due to the aversive impact of conflict, per se. Here we test this possibility using a task-switching paradigm known for its ability to show effects of prior inhibition above and beyond any effects of conflict.

\section{Conflict and inhibition during task switching}

When switching from one cognitive task to another, representations of the goals, stimuli, motor-responses and other aspects of the now-irrelevant task (i.e., the task-set, Monsell, 1996) can interfere with the ability to represent the goals, stimuli, motor-responses and other aspects of the newly-relevant task (for reviews see Kiesel et al., 2010; Monsell, 2003). Cognitive-control mechanisms are thought to help to minimize conflict-related decrements in speed and accuracy of task performance by (among other things) inhibiting the representations associated with the abandoned task set (for review see Koch, Gade, Schuch, \& Philipp, 2010). However, while inhibition is thought to be critical for minimizing interference with current task performance, it also appears to have lingering effects that impact performance should an individual have to 
return to and perform a previously-abandoned task at a later time (e.g., Arbuthnott \& Frank, 2000; Mayr \& Keele, 2000). Indeed, advances in the development of task-switching experimental procedures, such as Mayr and Keele's (2000) ABA vs. CBA task-sequence paradigm have allowed researchers to identify and dissociate these lingering effects of prior inhibition from other immediate effects of stimulus- or response-related conflict during taskswitching (e.g., Costa \& Friedrich, 2012). We capitalized on this by using a modified version of an ABA vs. CBA task-sequence paradigm that requires participants to switch between three visual tasks to identify and dissociate any lingering effects of prior inhibition on subsequent stimulus evaluations from any recent affective consequences of conflict.

\section{Current study}

On each trial of our experiment, participants searched for and made a visual discrimination about the odd-item-out in an array of four abstract art-like patterns. Each odd-item stood out relative to the other search items in the size (small or large), colour (yellow or green), or shape (circles or squares) of its constituent elements. Thus, each trial involved performing one of three different visual tasks — size discrimination, colour discrimination, or shape discrimination — which allowed us to form trial sequences that required participants to switch from one task to another in different orders. In 'ABA' sequences, participants on the first two trials of the sequence performed two different tasks (e.g., Task A = shape discrimination, then Task $\mathrm{B}=$ colour discrimination) and then on the third trial they performed the same task as they did on the first trial of the sequence (e.g., back to Task $\mathrm{A}=$ shape discrimination). In ' $\mathrm{CBA}$ ' sequences, participants performed a different task on each trial (e.g., Task $\mathrm{C}=$ size discrimination, then Task $\mathrm{B}=$ colour discrimination, and then Task $\mathrm{A}=$ shape discrimination).

The key measures of interest in such ABA vs. CBA task-sequence paradigms concerns response speed and accuracy on the third trial of each sequence, with the standard finding that 
performance is worse on the third trial of ABA sequences than on CBA sequences (Mayr \& Keele, 2000) because of having to return to a task and overcome the inhibition that was previously applied to its task-related representations when it was abandoned n-2 trials before. This n-2 repetition cost—-slower, less accurate responses — is what Mayr \& Keele (2000) termed the backward inhibition effect. A key feature of their paradigm is that the final trials on ABA and CBA sequences are exactly the same aside from whether the task $n-2$ trials before is repeated (ABA) or switched (CBA). Both types of sequences end on Task A, so involve the same task/stimuli/responses on the final trial. And the final trial in both sequences (Task A) requires switching from the same preceding task (Task B), with the same level of conflict that this may create. Moreover, the resulting backward inhibition effect on performance occurs even when other obvious sources of conflict, such as that from multiple competing stimulus- or responserelated representations, is minimized (Costa \& Friedrich, 2012).

Our main innovation was to combine each ABA and CBA task-switching sequence with an affective-evaluation procedure in which participants rated the emotional tone of the item they had just responded to in the third trial of the immediately-preceding sequence. While we expected to replicate the backward inhibition effect — the standard increase in response latency and errors on the third trial of the ABA sequences relative to those on CBA sequences - we were particularly interested in participants' subsequent affective reactions to the odd-one-out items from those trials. If inhibition has negative affective consequences for associated stimuli that exceed any aversive effects of conflict, then ABA items should receive more negative ratings than CBA items. If the stimulus-devaluation effects previously attributed to inhibition are instead due to the aversive impact of conflict, per se, then ABA items and CBA items should be equally disliked because of the equivalent levels of conflict that are created with the two task-switching sequences. 
To assess whether any affective consequences of backward inhibition vary with differences in general level of conflict, we also performed a between-subjects manipulation of the overall level of task-switching conflict by limiting participants' ability to predict the order of tasks that would have to perform (Unpredictable-order Group) or by providing a perfectlypredictable cue about the upcoming order of tasks prior to each sequence (Predictable-order Group). As a further test of the hypothesis that inhibition has negative affective consequences for associated stimuli over and above any aversive effects of conflict, we also examined whether fluctuations in the main behavioural index of backward inhibition-slowed response timeswere linked to the magnitude of affective devaluation of ABA items.

To anticipate our results, we observed standard backward inhibition effects on reaction time and accuracy, as well as affective devaluation of ABA stimuli relative to ratings of CBA stimuli. This devaluation effect was not significantly impacted by task-order uncertainty. Moreover, the response-time index of inhibition was larger on ABA trials in which the associated stimuli were later rated negatively than on trials preceding relatively positive ratings, suggesting a link between inhibition and the coding of stimulus value in the task-switching domain.

\section{Methods}

The methods reported here are a modified version of those used by Mayr \& Keele (2000) and Costa \& Friedrich (2012). All materials and procedures were approved by the Research Ethics Board at the University of Guelph (REB protocol \#17-10-103).

\section{Participants}

A total of 99 University of Guelph undergraduate students from the Psychology researchparticipant pool participated in exchange for course credit. All participants provided informed consent; all had normal or corrected-to-normal vision. One participant reported having a 
neurological disorder, and another failed to provide rating responses on the majority of trials, so their data were excluded from analyses. Participants were assigned to one of two separate groups. The desired sample size for each group was guided by a power analysis performed using G*Power (Faul et al., 2007), which indicated that 54 participants would be required to detect a similar-sized effect on stimulus ratings $(\mathbf{d z}=.5)$ as in prior cognitive-behavioural investigations of the devaluation-by-inhibition hypothesis (e.g., Clancy et al., 2019; Fenske et al., 2004; Ferrey et al., 2012; Raymond et al., 2005) with a power of .95 at an alpha of .05. We exceeded this slightly with the Unpredictable-order Group $(\mathrm{N}=56$; age: $M=18.6$ years, $S D=0.9 ; 43$ females, 13 males), but fell short with the Predictable-order Group $(\mathrm{N}=41$; age: $M=19.1$ years, $S D=$

$3.4 ; 32$ females, 9 males) due to restrictions on in-person data collection due to the SARS-CoV-2 global pandemic.

\section{Stimuli and apparatus}

Stimuli were abstract art-like Mondrian patterns that were created using a script in PsychoPy2 (Pierce et al., 2019) that randomly generated overlapping shapes within a larger square border that subtended approximately $4.65^{\circ}$ visual angle at a viewing distance of approximately $70 \mathrm{~cm}$. While each Mondrian was a unique pattern, the size, colour, and shape of its internal elements were constrained to form one of six types of images, each used in only one of the three different tasks. Specifically, the elements of the Mondrians for each task were: large or small blue-hued squares for the Size task, green-hued or yellow-hued triangles for the Colourtask, and squares or circles in red hues for the Shape task (see Figure 1). Five hundred seventyeight Mondrians of each type were used for a total of 3,468 stimuli. Stimulus presentation and behavioural-response collection were controlled using PsychoPy2 software (Pierce et al., 2019) running on an Intel-based personal computer with a $68.6 \mathrm{~cm}$ LCD monitor (resolution: $2560 \mathrm{x}$ 1440 pixels) and a standard computer keyboard and mouse. 
Task-switching sequences: Odd-item-out visual search trials

ABA
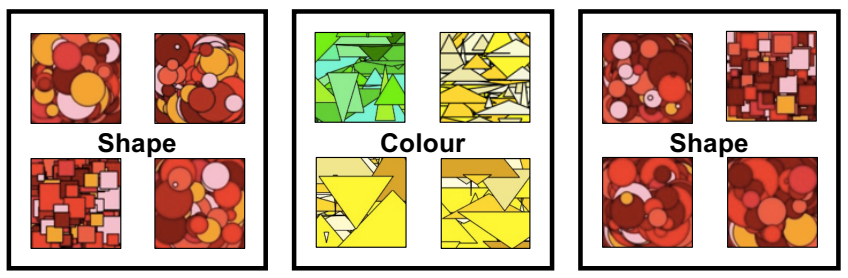

CBA

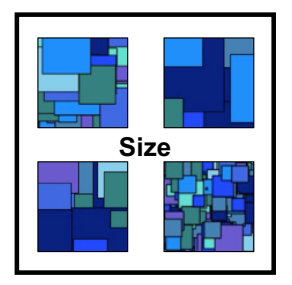

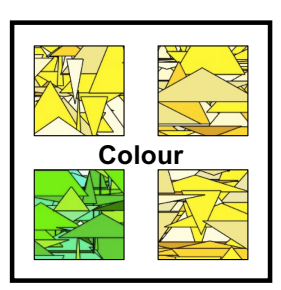

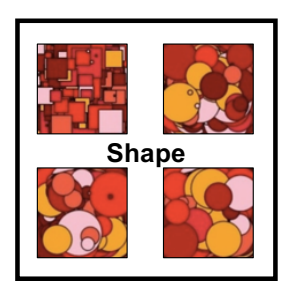

Affective-evaluation: How cheerful?

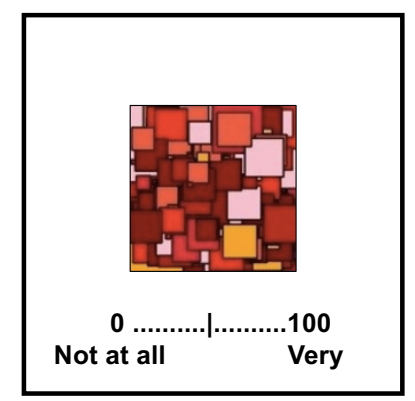

Figure 1. Examples of stimuli and order of events for ABA and CBA task-switching sequences. Each sequence consisted of three odd-one-out visual search trials, in which participants looked for the Mondrian pattern whose elements had a unique shape, unique colour, or unique size. ABA sequences contained a n-2 task repetition, whereas CBA contained a n-2 task switch. After each task-switching sequence, participants rated the emotional tone $(0=$ 'not at all cheerful' to $100=$ 'very cheerful') of the Mondrian stimulus that had just appeared as the odd-one-out on the final visual-search trial of that sequence.

\section{Design \& Procedure}

Our experimental approach involved combining ABA and CBA task-switching sequences of odd-one-out visual search trials with an affective-evaluation procedure in which participants rated the emotional tone of the critical stimulus from the final trial in each task-switching sequence. The resulting mixed-factors experimental design included Sequence (ABA, CBA) as a within-subjects factor and Group (Unpredictable-order, Predictable-order) as a between-subjects factor. The experiment began with a block of 48 practice trials in which participants learned how to perform each of the three different visual-search tasks (16 consecutive trials per task, with the order of tasks randomly determined for each participant). This was followed by four blocks of 48 
task-switch plus affective-evaluation sequences (192 total). Each experimental block contained an equal number of $\mathrm{ABA}$ and CBA sequences. The order of sequences and order of tasks within each sequence was otherwise randomized. For the Unpredictable-order Group, this meant that participants had no advance knowledge about which task (and thus which stimuli/responses) to expect from one trial to another. The Predictable-order Group, in contrast, was provided this advance information by a precue before each task-switching sequence that indicated the exact order of the next three tasks. Participants were allowed to rest between blocks as long as needed. The entire experiment took approximately 45 minutes to complete.

ABA and CBA task-switching sequences. Each task-switching sequence consisted of three odd-one-out visual search trials. The order of tasks in each trial sequence was randomized with the constraint that each possible task order occurred an equal number of times throughout the experiment, and that half of the trial orders formed ABA sequences that contained a n-2 task repetition (e.g., shape, colour, shape) and the other half formed CBA sequences that contained a n-2 task switch (e.g., size, colour, shape). Whereas the Unpredictable-order participants were not shown a precue display before task-switching sequences, the Predictable-order participants were shown a $3000 \mathrm{~ms}$ precue made of three words denoting the tasks (e.g., "Size Colour Shape") in the upcoming sequence. This precue appeared at display center and subtended approximately $0.5^{\circ} \times 12^{\circ}$ visual angle. For both groups, each odd-one-out visual search trial began with a 500 ms fixation cross at display centre, followed by a search array, which remained visible until response.

Each search array contained four Mondrian stimuli presented at the corners of an imaginary square, such that each Mondrian was centred $4.65^{\circ}$ above or below and $4.65^{\circ}$ to the left or right of fixation. Three of the Mondrians in each search array contained the same taskspecific feature and another (the odd-one-out) contained the other feature specific to that task. 
The odd-one-out stimulus was always a unique item (96 of each stimulus type; 576 total). The other three stimuli in each search array were randomly selected from a subset of unique images (255 of each stimulus type; 1530 total) and were allowed to repeat only after all 255 of the image-subset for that stimulus type had been presented. The positions of the odd-one-out and other three stimuli were randomly determined for each array, with the constraint that each type of stimulus appeared as the odd-one-out in each array position an equal number of times throughout the experiment.

Participants were asked to find the odd-one-out 'singleton' on each trial and then indicate its stimulus type as quickly as they could without making mistakes using the key assigned to that stimulus type. Each response key had a sticker on it that indicated its assigned stimulus type (i.e., Size-task keys: 'c' had a small blue-square sticker, 'v' had a large blue-square sticker; Colourtask keys: 'n' had a yellow-triangle sticker, 'm' had a green-triangle sticker; Shape-task keys: 'g' had a red-square sticker, ' $h$ ' had a red-circle sticker). Participants were asked to place their response finger on the ' $b$ ' key (blank sticker) between trials, which provided a central rest position that was approximately equidistant from the response keys. Although the type of stimuli in each search array clearly indicated which task was required, participants were also told which task was required on that trial by having its name ("Size", "Colour", or "Shape") subtending approximately $0.5^{\circ} \times 3^{\circ}$ visual angle) appear at the center of the search array.

Affective evaluation procedure. At the end of each task-switching sequence, participants were shown the Mondrian stimulus that had just appeared as the odd-one-out on the final visual-search trial of the preceding sequence. Because each of the odd-one-out items was unique, so too was each of the to-be-rated items. Participants rated the emotional tone of the item (How cheerful?) using the computer mouse by moving the slider on a scale (from $0=$ 'not at all cheerful' to $100=$ 'very cheerful') that appeared directly underneath the Mondrian, and then 
clicking a 'submit' button immediately below the scale. Participants were instructed that they needed to first move the slider before clicking the 'submit' button or else the experiment would advance without recording a rating response for that trial. Each affective-evaluation trial began with a $500 \mathrm{~ms}$ fixation cross at display centre followed by the to-be-rated stimulus, which remained visible until response.

\section{Data Analysis}

Our main measures of interest for the two experimental groups concerned behavioural performance (average correct response time and accuracy) for the final visual-search trial of ABA and CBA task-switching sequences, as well as the subsequent affective evaluations of the odd-item-out stimuli from those final visual-search trials. Prior to analysis we therefore calculated participant averages in each condition for each of these measures. For response times, only correct-response trials were considered and outliers in each task- and sequence-specific cell were removed prior to analysis using Van Selst \& Jolicoeur's (1994) recursive procedure. This resulted in the exclusion of $3.0 \%$ of trials. For affective ratings, only correct-response trials were considered because errors are known to have their own negative affective consequences (Chetverikov, 2014; Chetverikov, Jóhannesson, \& Kristjánsson, 2015). Participant averages for each of our measures of interest were then submitted to mixed-factors ANOVAs that included Sequence (ABA, CBA) as a within-subjects factor and Group (Unpredictable-order, Predictableorder) as a between-subjects factor.

If the level of inhibition indicated by relatively slowed response times on ABA trials is indeed linked to the affective devaluation of the associated Mondrian images, then fluctuations in the magnitude of response-time slowing should be linked to the magnitude of stimulus devaluation. We assessed this using a modified version of the approach developed by Kiss et al. (2007, 2008; see also Clancy, Fiacconi \& Fenske, 2019; De Vito, Al-Aidroos \& Fenske, 2017; 
Doallo et al., 2012) for investigating how neural and electrophysiological activity occurring during a task involving stimulus/response-inhibition can be predictive of subsequent affective ratings of the stimuli in that task. To examine whether fluctuations in the main behavioural index of backward inhibition —-slowed response times — were linked to the magnitude of affective devaluation of ABA items, we first sorted all of the ABA sequences into two categories for each participant based on whether they gave the final-trial image from each sequence a relatively negative rating (i.e., Low-rating: bottom $40 \%$ of trials with lowest ratings) or a relatively positive rating (i.e., High-rating: top $40 \%$ of trials with highest ratings). Average final-trial response times were calculated for each of these subsets of trials for each participant in each group. These averages were then submitted to a 2 (ABA-sequence item: Low-rating, High-rating) x 2 (Group) mixed-factors ANOVA. 


\section{Results}

\section{Behavioural performance results}

As shown in Figure 2, participants were slower and less accurate in their responses to the final visual-search trials of $\mathrm{ABA}$ sequences than they were for CBA sequences. These $\mathrm{n}-2$ repetition costs were significant for both response times $(\mathrm{RTs}), F(1,95)=31.87, p<.001, \eta^{2}{ }_{p}=$ .25 , and accuracy, $F(1,95)=8.41, p=.005, \eta^{2} p=.08$. This pattern of $\mathrm{n}-2$ repetition costs replicates the typical backward inhibition effect on behavioural performance.

Unpredictable-order

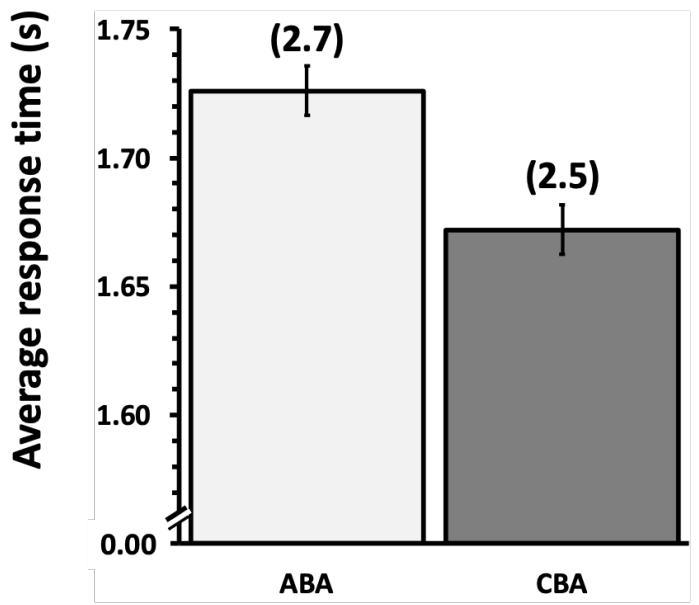

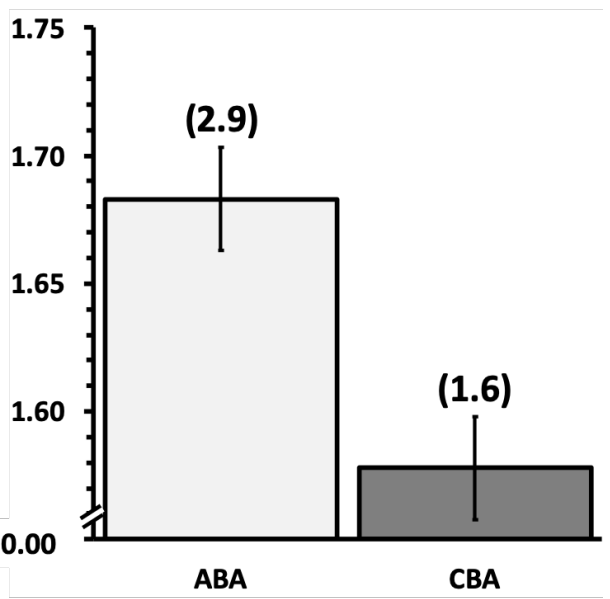

Task sequence

Figure 2. Behavioural performance on final trials of ABA and CBA task sequences for the Unpredictable-order group and the Predictable-order group. Average correct response times are shown in seconds with average percent errors in parentheses. Error bars represent within-subjects standard errors based on Loftus \& Masson's (1994) method.

There was neither a significant main effect of Group for RTs, $F(1,95)=.55, p=.46, \eta^{2} p=$ .006 . nor for accuracy, $F(1,95)=.44, p=.51, \eta^{2} p=.005$. There was also no significant Group $\mathrm{x}$ Sequence interaction for RTs - the primary behavioural index of backward inhibition - $F(1,95)$ $=3.12, p=.081, \eta^{2} p=.032$, although there was for accuracy, $F(1,95)=4.25, p=.042, \eta^{2} p=$ .043. Inspection of Figure 2 reveals that this was characterized by a larger backward inhibition effect on accuracy for the Predictable-order group (ABA minus CBA \% errors: $M=1.27 \%, S D=$ 
2.89) than for the Unpredictable-order group $(M=0.21 \%, S D=2.15)$.

\section{Affective-evaluation results}

As shown in Figure 3, final-trial Mondrian images received more negative ratings, overall, when they had just been encountered in ABA sequences than in CBA sequences, $F(1,95)$ $=8.72, p=.004, \eta_{p}^{2}=.084$. There was neither a significant main effect of Group, $F(1,95)=.29, p$ $=.59, \eta^{2}{ }_{p}=.003$, nor a significant Group x Sequence interaction, $F(1,95)=.43, p=.51, \eta^{2} p=$ .004 , for stimulus ratings. A planned contrast that focused solely on the Unpredictable-order group revealed that the difference in $\mathrm{ABA}$-item and $\mathrm{CBA}$-item ratings was significant under conditions in which the overall levels of conflict would have been highest due to task-order uncertainty, $t(55)=-2.24, p=.029$, Cohen's $d=-.30$. This pattern of results is consistent with the hypothesis that inhibition has negative affective consequences for associated stimuli that exceeds any emotional impact of conflict.

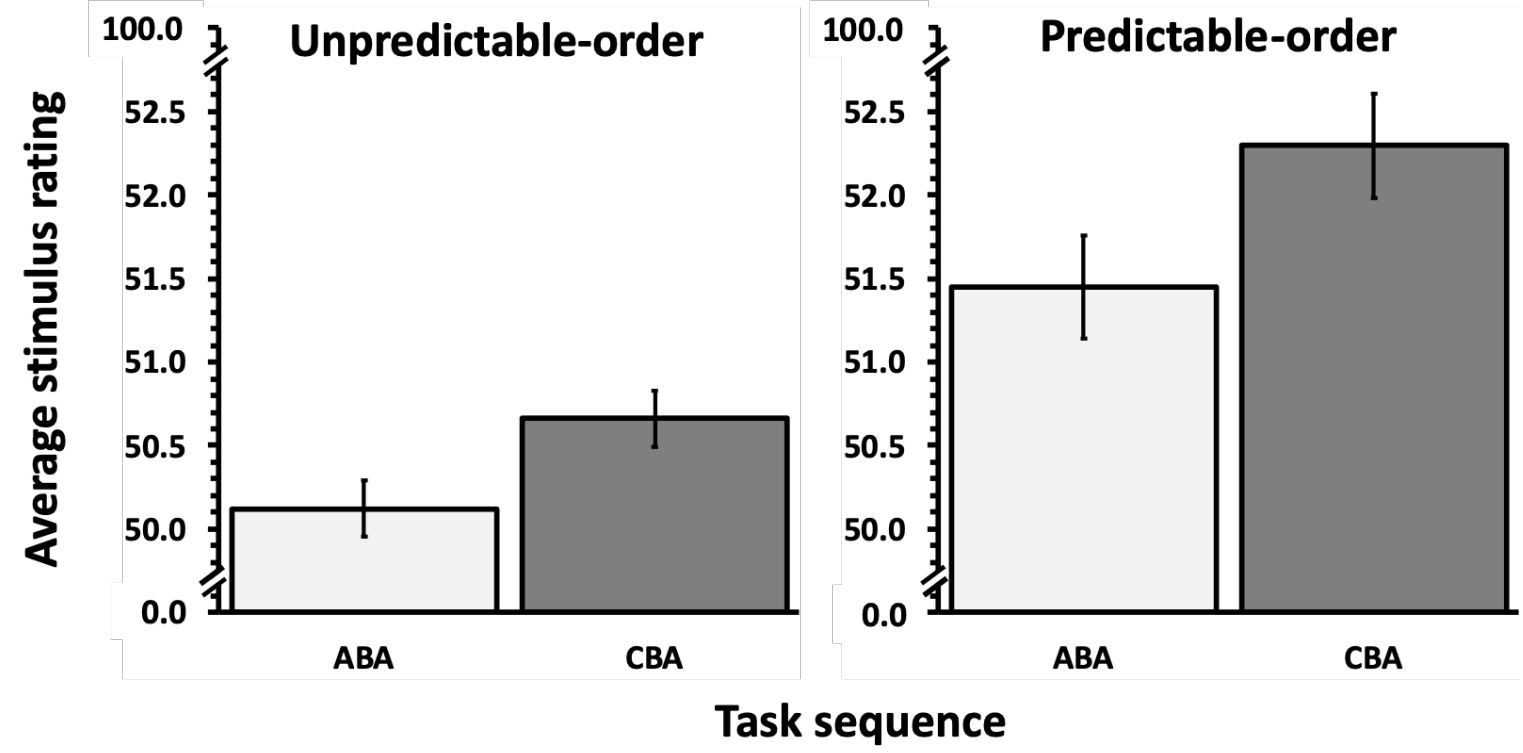

Figure 3. Average stimulus ratings (How cheerful? $0=$ not at all, $100=$ very) of Mondrian images from final trials of ABA and CBA task sequences. Error bars represent within-subjects standard errors based on Loftus \& Masson's (1994) method. 
Our analysis of whether ABA-trial performance was linked to subsequent stimulus ratings revealed that response times were significantly slower on Low-rating trials than on Highrating trials, $F(1,95)=5.31, p=.023, \eta^{2}{ }_{p}=.053$ (see Figure 4). In other words, there was a larger behavioural index of inhibition on ABA trials in which the associated stimuli were later rated negatively than on trials preceding positive ratings. This is consistent with the hypothesis that fluctuations in the magnitude of inhibition are linked to the magnitude of stimulus devaluation. Moreover, the magnitude of this difference was not significantly impacted by varying levels of conflict due to the difference in task-order uncertainty across the two groups, $F(1,95)=.12, p=$ $.73, \eta^{2}=.001$

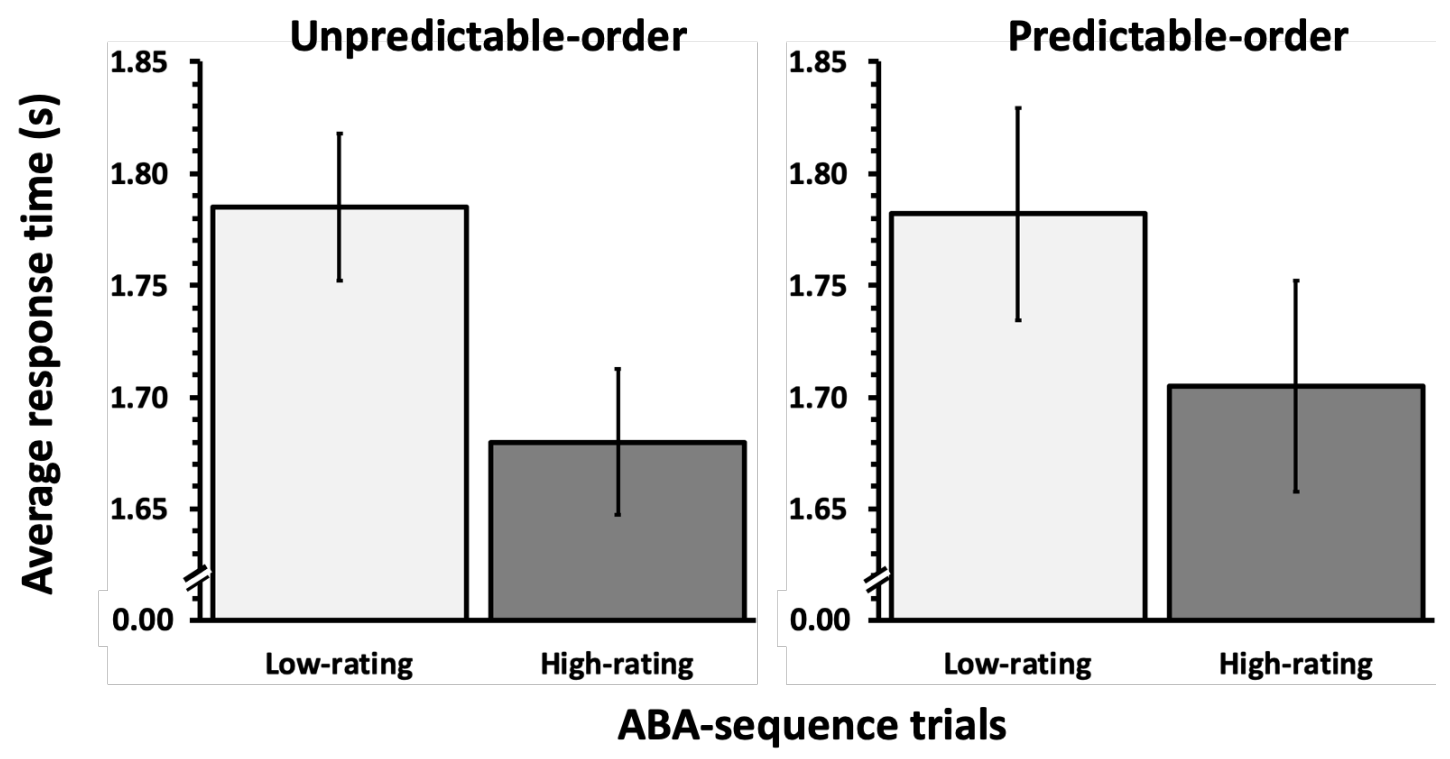

Figure 4. Average correct response times in seconds for the final trial of ABA sequences for the Unpredictable-order group and the Predictable-order group separated by subsequent affective rating (Low, High) of the Mondrian image from that trial. Error bars represent standard errors based on Loftus \& Masson's (1994) method for within-subjects designs. 


\section{Discussion}

Recent shifts in neurocognitive theory focus on conflict as a manifestation of the fundamental integration of emotion with cognitive-control (e.g., Botvinick, 2007; Dreisbach \& Fisher, 2015; Dignath et al., 2020; Inzlicht, Bartholow, \& Hirsh, 2015; Shackman et al., 2011). Combined with evidence that conflict between competing stimulus/response representations elicits negative affect and thereby leads to more negative evaluations of associated stimuli (Damen et al., 2018; Fritz \& Dreisbach, 2013; 2015; Goller et al., 2019), it makes sense that researchers (e.g., Chetverikov \& Kristjansson, 2015; 2016) would question whether stimulusdevaluation effects previously attributed to the affective consequences of inhibition are instead due to the emotional impact of conflict. Resolving whether inhibition has negative affective consequences that exceed any emotional impact of conflict is therefore important for the interpretation of a large and growing corpus of findings that have been thought to reflect stimulus devaluation by inhibition (e.g., Chen, Veling, Dijksterhuis, \& Holland, 2016; Chen, Holland, Quandt, Dijksterhuis, \& Veling, 2019; Clancy et al., 2019; De Vito \& Fenske, 2017; 2018; De Vito et al., 2017; 2018; Driscoll et al., 2018; 2020; Fenske et al., 2004; 2005; Ferrey et al., 2012; Frischen et al., 2012; Goolsby et al., 2009; Houben et al., 2012; Kihara et al., 2011; Kiss et al., 2007; 2008; Martiny-Huenger et al., 2014; Raymond et al., 2003; 2005; Vivas et al., 2016; Veling et al., 2008; 2013; Wessel \& O’Doherty, 2014; Wessel et al., 2015).

We chose to address this issue using Mayr and Keele's (2000) ABA vs. CBA taskswitching paradigm because of its well-established ability to show effects of prior inhibition above and beyond any effects of conflict. We found that $\mathrm{ABA}$ items subsequently received more negative ratings than $\mathrm{CBA}$ items, despite the equivalent levels of conflict that are created with these different task-switching sequences. This suggests that there is a link between inhibition and the coding of stimulus value in the task-switching domain that exceeds any emotional impact of 
conflict. We also found that the response-time index of inhibition was larger on ABA trials in which the associated stimuli were later rated negatively than on ABA trials preceding positive ratings. This latter finding converges with the results of prior studies using similar analyses of neural and electrophysiological correlates of inhibition (Clancy et al., 2019; De Vito et al., 2017; Doallo et al., 2012; Kiss et al. 2007; 2008) to suggest that trial-by-trial fluctuations in the level of inhibition applied during a prior task switch is linked to the subsequent level of affective devaluation of the associated stimuli.

Our findings also converge with other recent reports (Van Dessel, Liefooghe, De Houwer, 2020; Vermeylen, Braem, \& Notebaert, 2019) to underscore the potential usefulness of task-switching paradigms for further exploring links between emotion and cognitive control. Vermeylen et al. (2019), for example, provided the original demonstration that task-switching elicits negative affect. In their study, they assessed affective evaluations of cue words that indicated whether the participant would again perform a just-performed task or switch to a new task. They found that task-switch cues were evaluated as more negative than task-repeat cues. Van Dessel et al. (2020) replicated this effect and showed further that it emerges only after participants have had actual experience switching between and repeating different experimental tasks, but also generalizes to impact evaluations of previously-unseen stimuli when participants are told that these new stimuli will serve as new task-switch and task-repeat cues.

Our results expand on these prior task-switching affective findings by suggesting that the inhibition that is engaged to facilitate the switch from one task to another is a critical source of the negative affect elicited during task switches. The evidence that both switch-inducing cues (Vermeylen et al., 2019; Van Dessel et al., 2020) and specific stimuli related to a switched-from task are affectively devalued further suggests that the affective consequences of cognitivecontrol processes during task-switching impact multiple levels of neurocognitive representations. 
Indeed, one of the benefits of task-switching paradigms for future explorations of the links between emotion and cognitive control is the fact that there are so many different task-related aspects - goals, stimuli, motor-responses, etc. — that can be separately and jointly manipulated to investigate the characteristics and consequences of cognitive-affective processes across various levels of representation.

Our manipulation of general level of conflict via differences in task-order uncertainty did not lead to significant differences between the Unpredictable-order group and the Predictableorder group in our main response-time index of backward inhibition or in stimulus ratings, although it did impact the magnitude of n-2 repetition costs on final-trial response accuracy. While our experiment may have lacked sufficient statistical power for detecting between-group difference on the other measures, it is important to note that the ABA items were devalued relative to CBA items even for the Unpredictable-order group in which overall levels of conflict would have been highest.

We interpret our results as evidence that stimulus devaluation by inhibition can exceed any emotional impact of cognitive conflict. However, it is important to emphasize that this does not mean that the affective consequences of inhibition are entirely independent of conflict. In fact, the small subset of studies that have tried to assess how conflict and inhibition might be related in determining affective response have shown that the strongest negative reactions occur for the stimuli with the greatest potential to interfere with task performance, such as those nearest task-related targets (Martiny-Huenger et al., 2014; Raymond et al., 2005) or that are particularly salient because of prior exposure (Frischen et al., 2012) or their resemblance to task-related targets (De Vito et al., 2017). Such findings have been interpreted as confirming the reactive nature of inhibition (e.g., Houghton and Tipper, 1994) whereby increasing levels of interference potential require increasing levels of inhibition to suppress the corresponding stimulus 
processing or inappropriate motor-responses. This has led to theoretical accounts and experimental observations of stimulus-linked affective response that emphasize this inextricable link between conflict and inhibition (e.g., Ferrey, Burleigh, \& Fenske, 2015). Nevertheless, future work is needed to better understand exactly how conflict and inhibition are related and how they fit together within broader frameworks of the integration of emotion with cognitivecontrol. For now, our finding that the affective consequences of inhibition exceed any emotional impact of task-related conflict indicates that the affective-devaluation effects previously attributed to inhibition are not due to the emotional impact of conflict, per se.

\section{Open Practices Statement}

The data and materials for this study are available at https://osf.io/ekf2w/ 


\section{References}

Anderson, M. C., Bunce, J. G., \& Barbas, H. (2016). Prefrontal-hippocampal pathways underlying inhibitory control over memory. Neurobiology of Learning and Memory, 134, 145-161. https://doi.org/10.1016/j.nlm.2015.11.008

Arbuthnott, K., \& Frank, J. (2000). Executive control in set switching: Residual switch cost and task-set inhibition. Canadian Journal of Experimental Psychology, 54(1), 33-41. https://doi.org/10.1037/h0087328

Bari, A., \& Robbins, T. W. (2013). Inhibition and impulsivity: Behavioral and neural basis of response control. Progress in Neurobiology, 108, 44-79. https://doi.org/10.1016/j.pneurobio.2013.06.005

Botvinick, M. W. (2007). Conflict monitoring and decision making: Reconciling two perspectives on anterior cingulated function. Cognitive Affective \& Behavioral Neuroscience, 7, 356-366. https://doi.org/10.3758/CABN.7.4.356

Botvinick, M. M., Braver, T. S., Barch, D. M., Carter, C. S., \& Cohen, J. D. (2001). Conflict Monitoring and Cognitive Control. Psychological Review, 108(3), 624-652. https://doi.org/10.1037/0033-295X.108.3.624

Borst, J. P., Taatgen, N. A., \& van Rijn, H. (2010). The problem state: A cognitive bottleneck in multitasking. Journal of Experimental Psychology: Learning, Memory, and Cognition, 36(2), 363-382. https://doi.org/10.1037/a0018106

Chen, Z., Holland, R. W., Quandt, J., Dijksterhuis, A., \& Veling, H. (2019). When mere action versus inaction leads to robust preference change. Journal of Personality and Social Psychology. https://doi.org/10.1037/pspa0000158 
Chen, Z., Veling, H., Dijksterhuis, A., \& Holland, R. W. (2016). How does not responding to appetitive stimuli cause devaluation: Evaluative conditioning or response inhibition? Journal of Experimental Psychology: General, 145(12), 1687. https://doi.org/10.1037/xge0000236

Chetverikov, A. (2014). Warmth of familiarity and chill of error: Affective consequences of recognition decisions. Cognition \& Emotion, 28(3), 385-415. https://doi.org/10.1080/02699931.2013.833085

Chetverikov, A., \& Kristjánsson, Á. (2016). On the joys of perceiving: Affect as feedback for perceptual predictions. Acta Psychologica, 169, 1-10. https://doi.org/10.1016/j.actpsy.2016.05.005

Chetverikov, A., Jóhannesson, Ó. I., \& Kristjánsson, Á. (2015). Blaming the victims of your own mistakes: How visual search accuracy influences evaluation of stimuli. Cognition and Emotion, 29(6), 1091-1106. https://doi.org/10.1080/02699931.2014.968097

Clancy, E. M., Fiacconni, C. M., \& Fenske, M. J. (2019). Response inhibition immediately elicits negative affect and devalues associated stimuli: Evidence from facial electromyography. Progress in Brain Research, (247), 169-191. https://doi.org/10.1016/bs.pbr.2019.03.019

Costa, R \& Friedrich, F. (2012). Inhibition, Interference, and conflict in task-switching. Psychonomic Bulletin \& Review, 19(6), 1193-1201. https://doi.org/10.3758/s13423-012$\underline{0311-1}$

De Vito, D., Al-Aidroos, N., \& Fenske, M. J. (2017). Neural evidence that inhibition is linked to the affective devaluation of distractors that match the contents of working memory. Neuropsychologia, 99, 259-269. https://doi.org/10.1016/j.neuropsychologia.2017.03.022 
De Vito, D., \& Fenske, M. J. (2017). Suppressing memories of words and familiar objects results in their affective devaluation: Evidence from Think/No-think tasks. Cognition, 162, 1-11. https://doi.org/10.1016/j.cognition.2017.01.020

De Vito, D., Ferrey, A. E., Fenske, M. J., \& Al-Aidroos, N. (2018). Cognitive-behavioral and electrophysiological evidence of the affective consequences of ignoring stimulus representations in working memory. Cognitive, Affective, \& Behavioral Neuroscience, 18(3), 460-475. https://doi.org/10.3758/s13415-018-0580-x

Dignath, D., Eder, A. B., Steinhauser, M., \& Kiesel, A. (2020). Conflict monitoring and the affective-signaling hypothesis - an integrative review. Psychonomic Bulletin \& Review, 1-68. Advance online publication. https://doi.org/10.3758/s13423-019-01668-9

Di Lemma, L. C. G., \& Field, M. (2017). Cue avoidance training and inhibitory control training for the reduction of alcohol consumption: a comparison of effectiveness and investigation of their mechanisms of action. Psychopharmacology, 234(16), 2489-2498. https://doi.org/10.1007/s00213-017-4639-0

Doallo, S., Raymond, J. E., Shapiro, K. L., Kiss, M., Eimer, M., \& Nobre, A. C. (2012). Response inhibition results in the emotional devaluation of faces: Neural correlates as revealed by fMRI. Social Cognitive and Affective Neuroscience. https://doi.org/10.1093/scan/nsr031

Dreisbach, G., \& Fischer, R. (2012). Conflicts as aversive signals. Brain and Cognition, 78, 9498. https://doi.org/10.1016/j.bandc.2011.12.003

Driscoll, R. L., Clancy, E. M., \& Fenske, M. J. (2020, April 13). Response inhibition negatively impacts social-emotional evaluations of specific individuals. https://doi.org/10.31234/osf.io/f8jsc 
Driscoll, R. L., de Launay, K. Q., \& Fenske, M. J. (2018). Less approach, more avoidance: Response inhibition has motivational consequences for sexual stimuli that reflect changes in affective value not a lingering global brake on behavior. Psychonomic Bulletin and Review, 25(1), 463-471. https://doi.org/10.3758/s13423-017-1291-y

Fenske, M. J., Raymond, J. E., \& Kunar, M. a. (2004). The affective consequences of visual attention in preview search. Psychonomic Bulletin \& Review, 11(6), 1055-1061. https://doi.org/10.3758/BF03196736

Ferrey, A.E., Burleigh, T.J., Fenske, M.J., 2015. Stimulus-category competition, inhibition, and affective devaluation: a novel account of the uncanny valley. Frontiers in Psychology, 6, 1-15. http://dx.doi.org/10.3389/fpsyg.2015.00249

Ferrey, A. E., Frischen, A., \& Fenske, M. J. (2012). Hot or not: Response inhibition reduces the hedonic value and motivational incentive of sexual stimuli. Frontiers in Psychology, 3(DEC), 1-7. https://doi.org/10.3389/fpsyg.2012.00575

Frischen, A., Ferrey, A. E., Burt, D. H. R., Pistchik, M., \& Fenske, M. J. (2012). The affective consequences of cognitive inhibition: Devaluation or neutralization?. Journal of Experimental Psychology: Human Perception and Performance, 38(1), 169-179. https://doi.org/10.1037/a0025981

Fritz, J., \& Dreisbach, G. (2013). Conflicts as aversive signals: Conflict priming increases negative judgments for neutral stimuli. Cognitive, Affective, \& Behavioral Neuroscience, 13(2), 311-317. https://doi.org/10.3758/s13415-012-0147-1

Fritz, J., \& Dreisbach, G. (2015). The Time Course of the Aversive Conflict Signal. Experimental Psychology, 62(1), 30-39. https://doi.org/10.1027/1618-3169/a000271 
Gaspelin, N., \& Luck, S. J. (2018). The role of inhibition in avoiding distraction by salient stimuli. Trends in Cognitive Sciences, 22(1), 79-92. https://doi.org/10.1016/j.tics.2017.11.001

Gollwitzer, P. M., Martiny-Huenger, T., \& Oettingen, G. (2014). Affective consequences of intentional action control. In Advances in Motivation Science (Vol. 1, pp. 49-83). Academic Press.

Goolsby, B. A., Shapiro, K. L., \& Raymond, J. E. (2009). Distractor devaluation requires visual working memory. Psychonomic Bulletin and Review, 16(1), 133-138. https://doi.org/10.3758/PBR.16.1.133

Houben, K., Havermans, R. C., Nederkoorn, C., \& Jansen, A. (2012). Beer à No-Go: Learning to stop responding to alcohol cues reduces alcohol intake via reduced affective associations rather than increased response inhibition. Addiction, 107(7), 1280-1287. https://doi.org/10.1111/j.1360-0443.2012.03827.x

Houghton, G., \& Tipper, S. P. (1994). A model of inhibitory mechanisms in selective attention. In D. Dagenbach \& T. H. Carr (Eds.), Inhibitory processes in attention, memory, and language. San Diego, CA: Academic Press Inc..

Inzlicht M, Bartholow B. D., \& Hirsh J. B. (2015). Emotional foundations of cognitive control. Trends in Cognitive Science, 19(3), 126-132. https://doi.org/10.1016/j.tics.2015.01.004 Janczyk, M., \& Wühr, P. (2012). Inhibition does not always cause emotional devaluation. Experimental Psychology, 59(6), 372-378. https://doi.org/10.1027/1618-3169/a000168

Kiesel, A., Steinhauser, M., Wendt, M., Falkenstein, M., Jost, K., Philipp, A. M., \& Koch, I. (2010). Control and interference in task switching-A review. Psychological Bulletin, 136(5), 849-874. https://doi-org.subzero.lib.uoguelph.ca/10.1037/a0019842 
Kihara, K., Yagi, Y., Takeda, Y., \& Kawahara, J. I. (2011). Distractor devaluation effect in the attentional blink: Direct evidence for distractor inhibition, Journal of Experimental Psychology: Human Perception and Performance, 37(1), 168-179. https://doi.org/10.1037/a0019948

Kiss, M., Goolsby, B.A, Raymond, J.E., Shapiro, K.L., Silvert, L., Nobre, A.C., Eimer, M., 2007. Efficient attentional selection predicts distractor devaluation: Event-related potential evidence for a direct link between attention and emotion. Journal of Cognitive Neuroscience, 19 (8), 1316-1322. http://dx.doi.org/10.1162/jocn.2007.19.8.1316

Kiss, M., Raymond, J. E., Westoby, N., Nobre, A. C., \& Eimer, M. (2008). Response inhibition is linked to emotional devaluation: Behavioural and electrophysiological evidence. Frontiers in Human Neuroscience, 2, 1-9. http://dx.doi.org/10.3389/neuro.09.013.2008

Koch, I., Gade, M., Schuch, S., \& Philipp, A. (2010). The role of inhibition in task switching: A review. Psychonomic Bulletin \& Review, 17(1), 1-14. https://doi.org/10.3758/PBR.17.1.1

Martiny-Huenger, T., Gollwitzer, P. M., \& Oettingen, G. (2014). Distractor devaluation in a flanker task: Object-specific effects without distractor recognition memory. Journal of Experimental Psychology: Human Perception and Performance, 40(2), 613-625. https://doi.org/10.1037/a0034130

Mayr, U. \& Keele, A. W. (2000) Changing internal constraints on actions: The role of backward inhibition. Journal of Experimental Psychology: General, 129(1), 4-26. https://doi.org/10.1037/0096-3445.129.1.4

Monsell, S. (1996). Control of mental processes. In V. Bruce (Ed.), Unsolved mysteries of the mind: Tutorial essays in cognition (pp. 93-148). Erlbaum, Taylor, \& Francis.

Monsell, S. (2003). Task switching. Trends in Cognitive Sciences, 7(3), 134-140. https://doi.org/10.1016/S1364-6613(03)00028-7 
Munakata, Y., Herd, S. a., Chatham, C. H., Depue, B. E., Banich, M. T., \& O’Reilly, R. C. (2011). A unified framework for inhibitory control. Trends in Cognitive Sciences, 15(10), 453-459. https://doi.org/10.1016/j.tics.2011.07.011

Peirce, J., Gray, J. R., Simpson, S., MacAskill, M., Höchenberger, R., Sogo, H., ... \& Lindeløv, J. K. (2019). PsychoPy2: Experiments in behavior made easy. Behavior Research Methods, 51(1), 195-203. https://doi.org/10.3758/s13428-018-01193-y

Raymond, J. (2009). Interactions of attention, emotion and motivation. Progress in Brain Research. https://doi.org/10.1016/S0079-6123(09)17617-3

Raymond, J. E., Fenske, M. J., \& Westoby, N. (2005). Emotional devaluation of distracting patterns and faces: A consequence of attentional inhibition during visual search?. Journal of Experimental Psychology: Human Perception and Performance. https://doi.org/10.1037/0096-1523.31.6.1404

Shackman, A. J., Salomons, T. V., Slagter, H. A., Fox, A. S., Winter, J. J., \& Davidson, R. J. (2011). The integration of negative affect, pain and cognitive control in the cingulate cortex. Nature Reviews Neuroscience, 12, 154-167. https://doi.org/10.1038/nrn2994

Van Dessel, P., Liefooghe, B., De Houwer, J. (2020). The instructed task-switch evaluation effect: Is the instruction to switch tasks sufficient to dislike task switch cues?. Journal of Cognition, 3(1), pp. 1-13. https://doi.org/10.5334/joc.90

Veling, H., Aarts, H., \& Stroebe, W. (2013). Stop signals decrease choices for palatable foods through decreased food evaluation. Frontiers in Psychology, 4, 875. https://doi.org/10.3389/fpsyg.2013.00875 
Veling, H., Holland, R. W., \& van Knippenberg, A. (2008). When approach motivation and behavioral inhibition collide: Behavior regulation through stimulus devaluation. Journal of Experimental Social Psychology, 44(4), 1013-1019. https://doi.org/10.1016/j.jesp.2008.03.004

Vermeylen, L., Braem, S., \& Notebaert, W. (2019). The affective twitches of task switches: task switch cues are evaluated as negative. Cognition, 183, 124-130. DOI: https://doi.org/10.1016/j.cogni-\%20tion.2018.11.002

Vivas, A. B., Marful, A., Panagiotidou, D., \& Bajo, T. (2016). Instruction to forget lead to emotional devaluation. Cognition, 150, 85-91. https://doi.org/10.1016/j.cognition.2016.02.005

Wessel, J. R., Tonnesen, A. L., \& Aron, A. R. (2015). Stimulus devaluation induced by action stopping is greater for explicit value representations. Frontiers in Psychology, 6, 1640, 110. https://doi.org/10.3389/fpsyg.2015.01640 